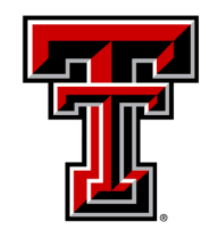

TEXAS TECH UNIVERSITY

Libraries"

\title{
DYNAMIC ANALYSIS OF MATERIAL EJECTION FROM CATHODIC METAL NANO-TIPS DUE TO LOCAL HEATING AND FIELD GENERATED STRESS
}

\section{The Texas Tech community has made this publication openly available. Please share how this access benefits you. Your story matters to us.}

\begin{tabular}{|c|c|}
\hline Citation & $\begin{array}{l}\text { X. Qiu and R. P. Joshi, "Dynamic Analysis of Material Ejection from } \\
\text { Cathodic Metal Nano-Tips Due to Local Heating and Field Generated } \\
\text { Stress," Physics of Plasmas 25, } 022109 \text { (2018). } \\
\text { https://doi.org/10.1063/1.5018441 }\end{array}$ \\
\hline Citable Link & https://hdl.handle.net/2346/86899 \\
\hline Terms of Use & $\begin{array}{l}\text { This article may be downloaded for personal use only. Any other } \\
\text { use requires prior permission of the author and AIP Publishing. }\end{array}$ \\
\hline
\end{tabular}




\section{Dynamic analysis of material ejection from cathodic metal nano-tips due to local heating and field generated stress}

Cite as: Phys. Plasmas 25, 022109 (2018); https://doi.org/10.1063/1.5018441

Submitted: 06 December 2017 . Accepted: 26 January 2018. Published Online: 09 February 2018

(D) X. Qiu, and R. P. Joshi

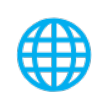

View Online

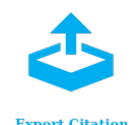

\section{ARTICLES YOU MAY BE INTERESTED IN}

Model evaluations of surface modification by energetic incident carbon atoms on graphene coated copper electrodes

Physics of Plasmas 26, 013501 (2019); https://doi.org/10.1063/1.5056766

Calculations of secondary electron yield of graphene coated copper for vacuum electronic applications

AIP Advances 8, 015325 (2018); https://doi.org/10.1063/1.5019360

Plasma parameters of the cathode spot explosive electron emission cell obtained from the model of liquid-metal jet tearing and electrical explosion

Physics of Plasmas 25, 053504 (2018); https://doi.org/10.1063/1.4999377 


\title{
Dynamic analysis of material ejection from cathodic metal nano-tips due to local heating and field generated stress
}

\author{
X. Qiu and R. P. Joshi ${ }^{\text {a) }}$ \\ Department of Electrical and Computer Engineering, Texas Tech University, Lubbock, Texas 79409, USA
}

(Received 6 December 2017; accepted 26 January 2018; published online 9 February 2018)

\begin{abstract}
The potential for explosive cathode emission due to nanoprotrusions subjected to Maxwell stress and heating from strong electric fields is probed self-consistently based on non-equilibrium moleculardynamics. The focus is on determining the electric field magnitudes that could lead to material ejection, assessing dependencies of the instability on the nanoprotrusion height and cross-sectional area, and the role of time-dependent thermal conductivity and local temperature changes. Our results indicate that large aspect ratios would facilitate mass ejection, with protrusion break up occurring over times in the $25 \mathrm{~ns}$ range, in agreement with experimental reports on explosive emission. Published by AIP Publishing. https://doi.org/10.1063/1.5018441
\end{abstract}

\section{INTRODUCTION}

Explosive emission cathodes, such as velvet, carbon fibers, ferroelectrics, metallic cathodes, and field-emitting arrays, are widely used for electron beam generation with current densities of over $100 \mathrm{~A} / \mathrm{cm}^{2}{ }^{1-3}$ Experiments have shown that the emitting surface of such cathodes presents a set of separately operating emitters. ${ }^{4}$ Mass ejection and plasma formation have both been reported. ${ }^{5-8}$ It has been confirmed that the underlying emission mechanism involves the explosions of micropoints on the cathode surface ${ }^{9,10}$ and appears to start from sites having large field enhancements or low local work functions. ${ }^{11,12}$ In order to obtain beams of high quality and tailor long lifetimes for explosive emission cathodes, it is necessary to first understand the mechanism of explosive emission, especially the initiation process. Simple, qualitative explanations for initiation center around the creation of high electric fields (in the $10^{7}-10^{8} \mathrm{~V} / \mathrm{cm}$ range) at cathode micro-protrusions ${ }^{13}$ which present sharp corners and edges for field enhancement. The high fields ${ }^{14,15}$ can then lead to electron emission and cause localized heating, perhaps even vaporization of the cathode material, ${ }^{16,17}$ along with the possible release of desorbed surface gases that could subsequently be ionized. ${ }^{10,18}$

Almost all previous calculations based on the field emission or thermal instability models at micropoints ${ }^{19-22}$ have relied on the hydrodynamic, continuum theory. Such approaches are likely inaccurate at nanoscales due to the breakdown of spatial averaging, the neglect of fluctuations and inherent noise, the inability to fold in details of the lattice structure and atomic arrangements, and their inability to adequately model finite size effects such as reductions in thermal conductivity upon spatial confinement. Furthermore, continuum models, regardless of the length scales, cannot effectively incorporate many of the material properties (such as the surface tension) that depend on the microscopic details of the local neighborhoods surrounding any location. The molecular dynamics approach, on the other hand, eliminates

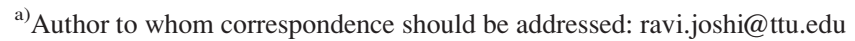

the above difficulties and provides a more accurate and fundamental analysis. In addition to heating, the existence of high electric fields is likely to produce strong Maxwell stress, which would exert pressure on the nanoprotruding material producing separation or ejection. This aspect has also generally been ignored in previous reports, though a recent analysis probed these issues based on molecular dynamics (MD) simulations. ${ }^{23}$

Here, we extend the recent calculations to include a selfconsistent, dynamic evolution of the internal temperatures. The important issue of material degradation at emitters observed in pulsed power systems at high electric fields, ${ }^{24}$ and the relatively fast time scale of this process, are probed. From a mechanical standpoint, the high electric fields can produce strong tensile Maxwell stresses at surfaces ${ }^{25}$ potentially leading to deformations or evaporative mass ejection; ${ }^{26-28}$ that typically occur over ultrashort (nanosecond) time scales. ${ }^{29}$ It might even be conjectured that localized heating due to electron emission could lead to an instability at the metal tip, similar to the Rayleigh-Plateau ${ }^{30,31}$ process for solid columns. ${ }^{32}$ Also, in this context, the lateral geometric confinement inherent in nanoprotrusions would shorten phonon mean free paths and reduce heat outflow, leading to decreases in the local thermal conductivity. ${ }^{33}$ The latter could then hasten temperature rise, and produce stronger and quicker thermally driven effects.

The present focus is on the following aspects: (a) determining the local electric field magnitudes that could lead to material ejection, (b) assessing dependencies of the instability on either the nanoprotrusion height or its cross-sectional area, (c) probing changes in the time scales with protrusion dimensions, and (d) conditions under which material melting leading to nanoprotrusion flattening rather than ejection, may occur. Such flattening would be a natural self-healing process.

\section{MODELING DETAILS}

The Maxwell stress on a nanoprotrusion can be calculated from knowledge of the surrounding electric field 
distribution. Experimental reports suggest fields as high as $10^{10} \mathrm{~V} / \mathrm{m}$ (and beyond) can exist under dc vacuum conditions in certain high power systems. ${ }^{34}$ Though the field distribution can easily be obtained for simple two-dimensional protrusions, ${ }^{14,35,36}$ here, a full three-dimensional approach based on COMSOL, a multi-physics software tool, was used. Knowledge of the field distribution then allows for calculations of the surface Maxwell stress as discussed previously. ${ }^{23}$ Information regarding the spatially varying Maxwell stresses was coupled into LAMMPS, a classical molecular dynamics (MD) simulator. ${ }^{37}$ As an illustrative example, a bias of 240 $\mathrm{V}$ between the nanostructure and a planar anode placed at a distance of $120 \mathrm{~nm}$ from the cathode substrate, resulted in very high fields on the order of $10 \mathrm{~V} / \mathrm{nm}$ at the nanoprotrusion edges, is given in Fig. 1. The resulting Maxwell pressure as shown qualitatively by the arrows was in the 515 to $555 \mathrm{MPa}$ range.

The MD technique essentially involves solving the equations of motion for interacting particles numerically, subject to appropriate initial and boundary conditions. ${ }^{38}$ The many-body embedded atom method (EAM) ${ }^{39}$ is used for the interaction potential between the cathode copper atoms. A consistent set of embedding functions and short-range repulsive pair interactions for the face-centered-cubic (FCC) metals (such as copper) is available to LAMMP users based on the data by Foiles et al. ${ }^{40}$ Results obtained from the MD simulations were visualized via the OVITO software. ${ }^{41}$ The simulation cell was taken to have a copper base (i.e., the substrate) on which the copper nanoprotrusion was erected as shown in the schematic of Fig. 2. The substrate dimensions were taken to be $10.845 \mathrm{~nm} \times 15.337 \mathrm{~nm} \times 3.067 \mathrm{~nm}$, while the free-standing column dimensions were varied to probe the effects of choosing different aspect ratios. The nanoprotrusion position was taken to be at the substrate center. The substrate lateral dimensions chosen ensured sufficient spacing between the nanoprotrusion and the edges to minimize any edge proximity effects. Nonetheless as a test, four other simulations were carried out, though the results are not shown here, with the protrusion slightly shifted along the two lateral directions to probe possible deviations in the

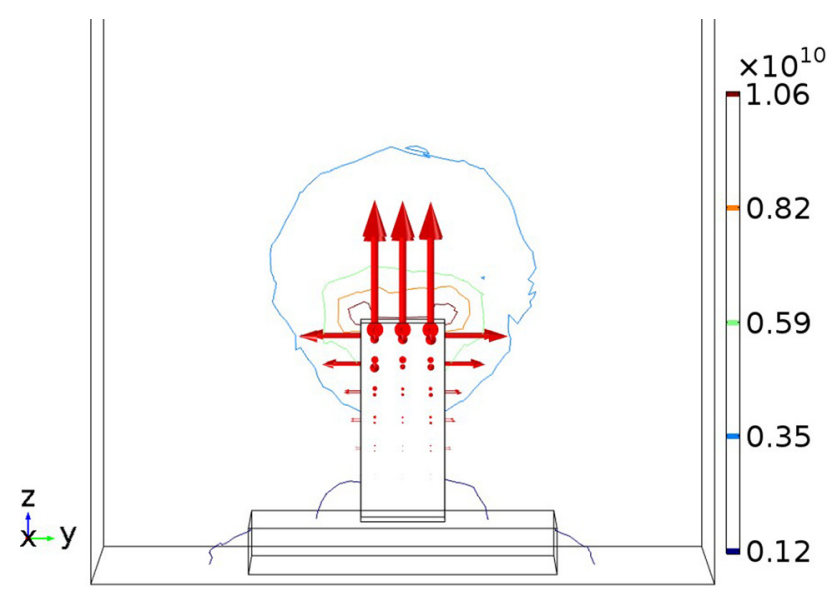

FIG. 1. Schematic showing the electric field contours and magnitude around a cathode nanoprotrusion and an anode placed at a distance of $120 \mathrm{~nm}$. The resulting Maxwell pressure was in the 515 to $555 \mathrm{MPa}$ range.

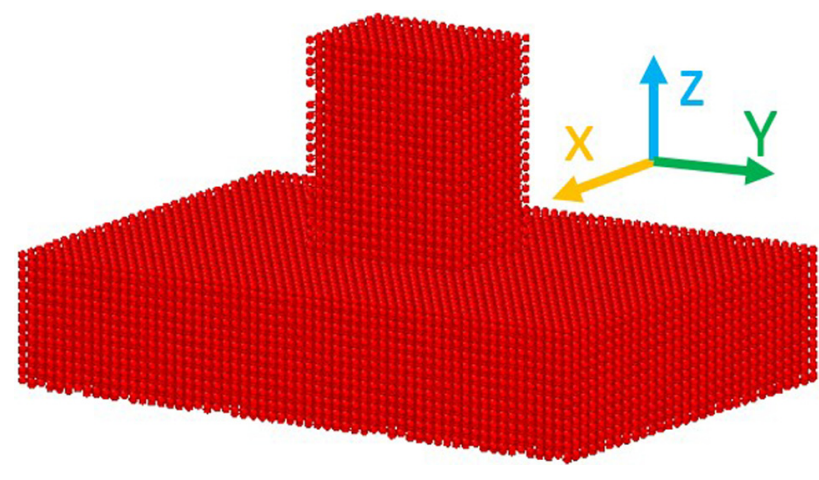

FIG. 2. Schematic showing the copper substrate and nanoprotrusion used for the molecular dynamics simulations.

results. The center of the nanoprotrusion was moved by $( \pm 0.5 \mathrm{~nm}, \pm 0.5 \mathrm{~nm})$ along the $x$ - and $y$-axes, respectively. However, the results for these four different positions remained nearly unchanged relative to the central location, in terms of the temperatures attained and time predicted for material ejection. Periodic boundary conditions were imposed along the $x$ - and $y$-directions. Atoms in the two lowest layers were fixed in position throughout the whole simulation, and a time-step of $1 \mathrm{fs}$ was used. The system was initially relaxed by running the LAMMPS simulations up to $150 \mathrm{ps}$ without any forces, to achieve thermodynamic equilibrium under the NpT isothermal-isobaric ensemble. Following energy minimization, atoms on the nanoprotrusion surfaces were subjected to Maxwell stresses, as computed from the COMSOL calculations, and NVE ensemble conditions were applied.

The temperature should be a function of position since effectively the present situation amounts to a "heating problem" with energy being added from the top end of the nanoprotrusion. This energy input would be the result of Joule heating at the emitting end, with the temperature gradually increasing over time throughout the entire structure. This was modeled by adding kinetic energy to the upper surface of the nanoprotrusion at a constant rate. The simulations were then run under the NVE ensemble that let the temperature grow throughout the spatial domain, in response to the diffusive heat flow. The entire simulation region along the $z$ direction was evenly divided into twenty layers. The first layer was at the bottom and coincided with the substrate boundary, while the twentieth layer was at the top of the nanoprotrusion.

From the COMSOL results for the electric fields, charges were assigned to the surface atoms based on Gauss' law. Since the discretization used in the COMSOL calculations was larger than the inter-atomic distances, an average charge was smeared over all atoms contained within a grid cell leading to an effective jellium model for a metal. However, more sophisticated numerical methods of surface charge distribution have been discussed in the literature. ${ }^{42}$

\section{SIMULATION RESULTS}

The dependence of nanoprotrusion height, or more generally the aspect ratio, is an important consideration that 

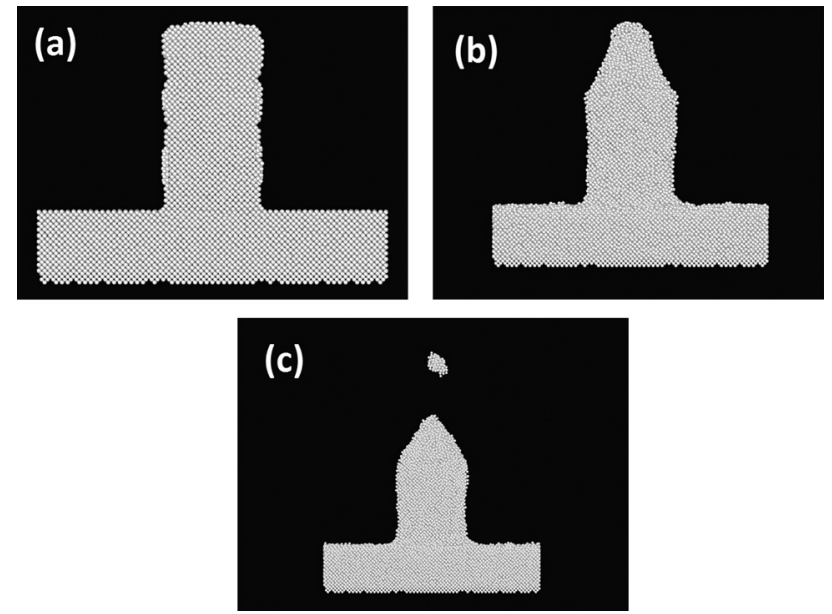

FIG. 3. Molecular dynamics simulation results for a $8.2 \mathrm{~nm}$ nanoprotrusion with self-consistent temperature evolution in an external electric field. The substrate dimensions were set at $10.845 \mathrm{~nm} \times 15.337 \mathrm{~nm} \times 3.067 \mathrm{~nm}$. (a) The initial $0 \mathrm{~ns}$ snapshot of the initial structure after energy minimization, (b) a 5.58 ns snapshot showing "necking" at the tip, and (c) a 5.696 ns snapshot showing material ejection from the cathode tip leaving behind a shorter protrusion

merits analyses given experimental observations of greater damage for the case of longer carbon fibers. ${ }^{24}$ This aspect was probed through the MD simulations. For example, in a study on carbon fiber cathodes that used both bimodal sample cathodes (consisting of $2 \mathrm{~mm}$ long fibers), and unimodal carbon fiber cathodes (consisting of shorter $1 \mathrm{~mm}$ fibers), structural damage was seen only in the longer $2 \mathrm{~mm}$ fibers. ${ }^{24}$ This result suggests a role for the aspect ratio, and the damage is likely to be more prevalent in longer structures.

Our MD simulations point to stronger and faster material ejection for taller nanoprotrusions. For example, snapshots at the time instants of $0 \mathrm{~ns}, 5.58 \mathrm{~ns}$, and $5.696 \mathrm{~ns}$ are shown for a $8.2 \mathrm{~nm}$ high structure in Figures 3(a)-3(c). The base area was chosen to be $8 \times 8$ lattice units, which corresponds to dimensions of $28.92 \AA$ by $40.9 \AA$. A narrowing or neck formation can be seen to form at the tip after about $5.58 \mathrm{~ns}$. As the narrowing progresses, the number of atoms in the constricted neighborhood reduces and so does the total binding force due to the combined intermolecular interactions. The simulation predicts complete mass ejection, leaving behind a shortened protrusion after about 5.696 ns. By contrast, a slightly taller $9.7 \mathrm{~nm}$ protrusion was predicted to undergo mass ejection at about $0.772 \mathrm{~ns}$ (results not shown), while the $10 \mathrm{~nm}$ case emitted copper molecules after just $0.118 \mathrm{~ns}$ as shown in Figs. 4(a)-4(d). The four snapshots in Figure 4 were obtained at the time instants of $0 \mathrm{~ns}, 97 \mathrm{ps}$, $117 \mathrm{ps}$, and $122 \mathrm{ps}$, respectively. An interesting aspect is that after the original tip is severed, successive puffs of ejected copper fragments are predicted in Fig. 4(d). Three factors contribute to this rapid release of additional copper material. First, the number of atoms in the neighborhood of the tip region is appreciably lowered after about 97 ps as seen from Fig. 4(b). This reduces the degree of inter-molecular binding and makes material ejection an easier process. Furthermore, heating at the top would be higher given the reduced lateral area for thermal transport, a factor that would augur well for faster and frequent fragmentation. Finally, the reduced diameter near the tip leads to greater instability. For example, metallic nanowires or nanoprotrusions are known to become unstable below a minimum diameter as the surface tension exceeds the maximum tension that could limit plastic flow. ${ }^{43}$ This critical diameter $d_{m}$ is roughly given by: $d_{m} \approx 2 \sigma_{S} / \sigma_{Y}$, where $\sigma_{S}$ is the surface tension and $\sigma_{Y}$ is the yield strength. For copper, using bulk values for these two quantities, yields a threshold estimate of $d_{m} \approx 15 \mathrm{~nm}$. Hence, nanoprotrusions smaller in diameter would be subject to an easier break-off.

A similar trend was obtained from simulations with a different base area of $9 \times 9$ lattice units $(=32.535 \AA \times 46.011 \AA$ ) .
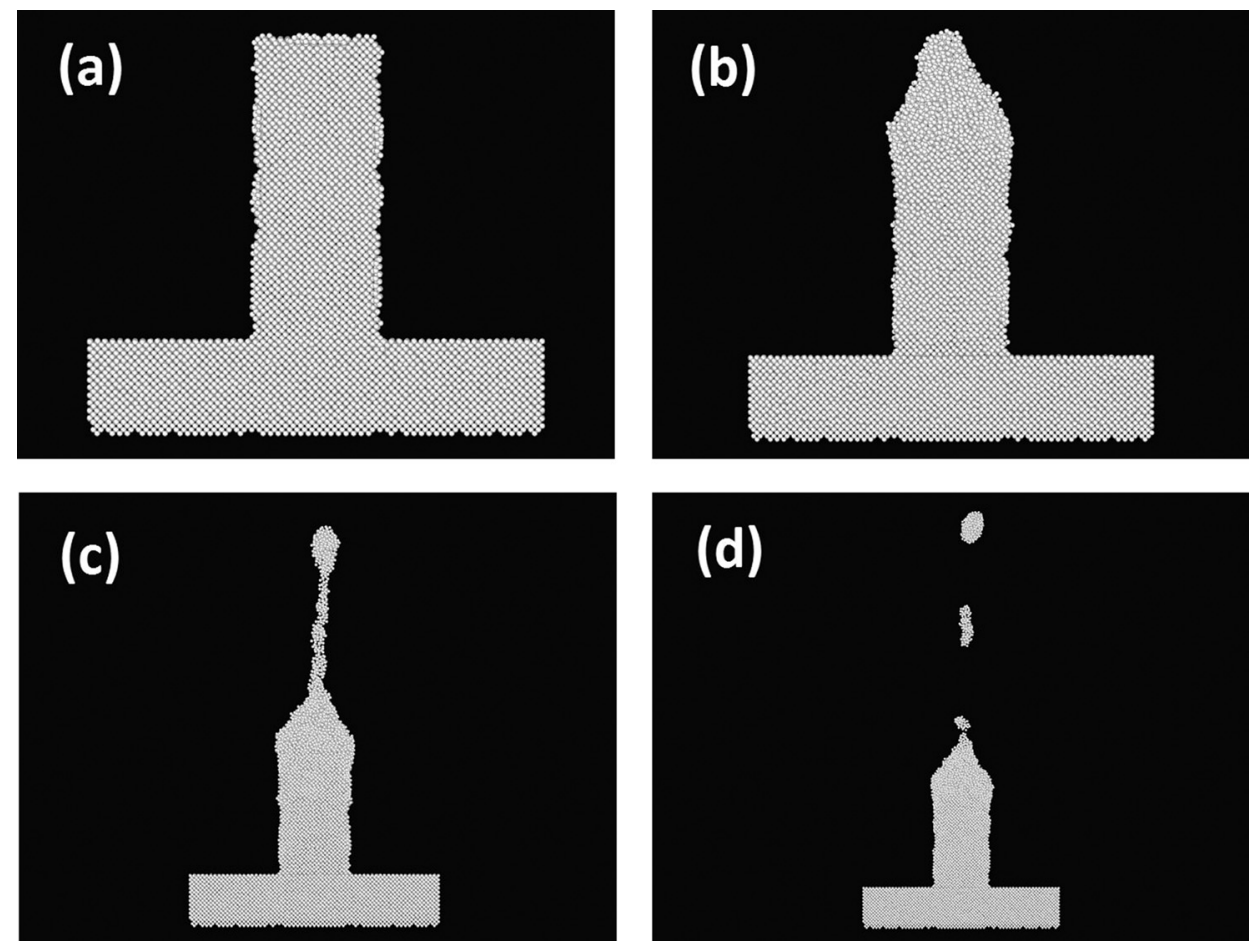

FIG. 4. Molecular dynamics simulation results for a taller $10 \mathrm{~nm}$ nanoprotrusion. As with Fig. 3, the substrate dimensions were $15.337 \mathrm{~nm}$ (width) and $3.067 \mathrm{~nm}$ (height). (a) The initial $0 \mathrm{~ns}$ snapshot of the initial structure after energy minimization, (b) A 97 ps snapshot showing "necking" at the tip, (c) the situation at $117 \mathrm{ps}$ almost at the breaking point, and (d) a 122 ps snapshot showing material ejection from the cathode tip. 
TABLE I. Mass ejection times for different protrusion heights and base dimensions.

\begin{tabular}{lcc}
\hline \hline Base size (lattice units) & Height $(\mathrm{nm})$ & Ejection time $(\mathrm{ns})$ \\
\hline $8 \times 8$ & 8.2 & 5.696 \\
$8 \times 8$ & 9.7 & 0.772 \\
$8 \times 8$ & 10 & 0.118 \\
$9 \times 9$ & 9.7 & 20.136 \\
$9 \times 9$ & 10 & 11.414 \\
$9 \times 9$ & 11 & 2.236 \\
$10 \times 10$ & 12 & 6.264 \\
$11 \times 11$ & 11 & 18.512 \\
$12 \times 12$ & 13 & 21.156 \\
\hline \hline
\end{tabular}

Though figures at different times for material ejection are not shown, various cases were also analyzed and the corresponding ejection times obtained. A summary of the ejection times based on the MD simulations is provided in Table I for different protrusion heights and base areas. An important outcome is the role of protrusion base area in influencing the material ejection time. For example, a slight increase from a base size of $8 \times 8$ lattice units to $9 \times 9$ units for a $9.7 \mathrm{~nm}$ tall protrusion is predicted to change the ejection time from $0.772 \mathrm{~ns}$ to $20.136 \mathrm{~ns}$. This is about an order of magnitude variation. These results are again in keeping with the thesis of faster and easier mass ejection from taller and thinner protrusions. Similarly, for a $10 \mathrm{~nm}$ height, the ejection time is predicted to change from about $0.118 \mathrm{~ns}$ for a base of $8 \times 8$ lattice units to $11.414 \mathrm{~ns}$ for a $9 \times 9$ base. A similar increase from $2.236 \mathrm{~ns}$ to $18.512 \mathrm{~ns}$ can be seen in Table I for a $11 \mathrm{~nm}$ tall protrusion in going from a $9 \times 9$ base to a $11 \times 11$ base. The results trend towards reductions in ejection time with increasing height, increased aspect ratio, and reduced base area.

However, the role of geometric dimensions on ejection is complex. At the simplest level, higher aspect ratios (i.e., either taller protrusions or smaller base area) lead to enhanced surface fields, ${ }^{15}$ and hence faster ejection times since the field-driven currents and corresponding dissipated power densities are increased. The basal area contributes to stability since the total (downward) stabilizing force on any atom in the surface layer depends on the number of neighboring atoms. For small cross-sections, this stability factor would scale roughly with area since distances between atoms would not vary appreciably, although the number of atoms contributing to binding would increase with area. However, for larger basal areas, though the number of interacting atoms increases, the larger distances of atoms further away lead to sub-linear contributions to the binding force. Another nuanced areal effect is the extent of the reduction in collective binding upon columnar necking. The start of even a small constriction can have a large fractional change in the adhesive force for small-area columns. Hence, any perturbative areal reductions for protrusions with a small base would likely move the system towards instability and disintegration, as obtained in the results of Table I. For example, at the $9.7 \mathrm{~nm}$ height, ejection time is predicted to decrease from $20.1 \mathrm{~ns}$ to $0.772 \mathrm{~ns}$ as the base area is reduced from $9 \times 9$ units to $8 \times 8$ units. A similar trend is seen for the $10 \mathrm{~nm}$ height with changes in the basal cross-section from $9 \times 9$ to

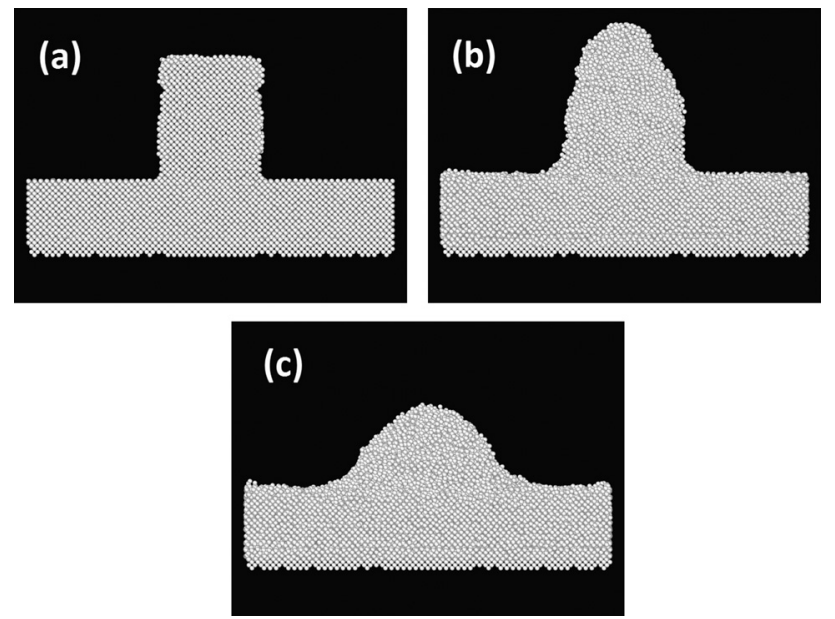

FIG. 5. Snapshots from the simulations for a much smaller $5.1 \mathrm{~nm}$ protrusion at: (a) the initial $\mathrm{t}=0$ instant, (b) a $5.5 \mathrm{~ns}$ time, and (c) the structure at $6.114 \mathrm{~ns}$ showing melting. As mentioned in the text, the substrate dimensions were set at $15.337 \mathrm{~nm}$ (width) and $3.067 \mathrm{~nm}$ (height).

$8 \times 8$ units. Finally, another geometric effect at play in the present case, and naturally folded into the MD calculation scheme, is the thermal conductivity reduction due to spatial confinement. Reductions in the lateral dimensions are known to decrease the thermal conductivity in free-standing structures due to relative increases in surface scattering. Consequently, regions close to the tip get far hotter in the case of narrow protrusions, thus increasing the kinetic energies of the atoms and making them more likely to escape binding. In any event, given these multitude of processes with complex dependencies on inter-atomic distances and their time evolution, it is difficult to construct simple scaling rules.

Three factors collectively contribute to the faster ejection times: (a) increases in the electric field as the aspect ratio becomes larger and more "needle-like." (b) Increases in the number of molecules interacting with each other within the nanoprotrusion for larger base areas leading to greater stability. (c) Slightly better heat conduction with increased area leading to lower rise in temperatures within the nanoprotrusion. The results of Table I show that ejection times on the order of $25 \mathrm{~ns}$ are possible for a base of $12 \times 12$ lattice units and a $13 \mathrm{~nm}$ height. This prediction is roughly in agreement with the experimental reports of explosive emission at cathode tips ${ }^{44}$ and also in line with the model predictions. ${ }^{29}$ Longer ejection times in the 50-100 ns range are easily conceivable for slightly larger base dimensions.

As a final comment on the height dependence, much shorter nanoprotrusions are not likely to fragment, but would likely melt due to local temperature increases and eventually smooth out over the substrate. This was observed in our simulations, with a typical result for a $5.1 \mathrm{~nm}$ tall protrusion shown in Fig. 5. The initial $(t=0)$ snapshot and an intermediate state at the $5.5 \mathrm{~ns}$ instant are shown in Figs. 5(a) and 5(b), while the snapshot after $6.114 \mathrm{~ns}$, is presented in Fig. 5(c). Lateral spreading and flattening is evident in Fig. 5(c). The time development is crudely analogous to the melting of a small ice-cream scoop on a hot day. Basically, for this small height, the intermolecular attractive forces are much stronger. Furthermore, the local temperatures at the tip do 

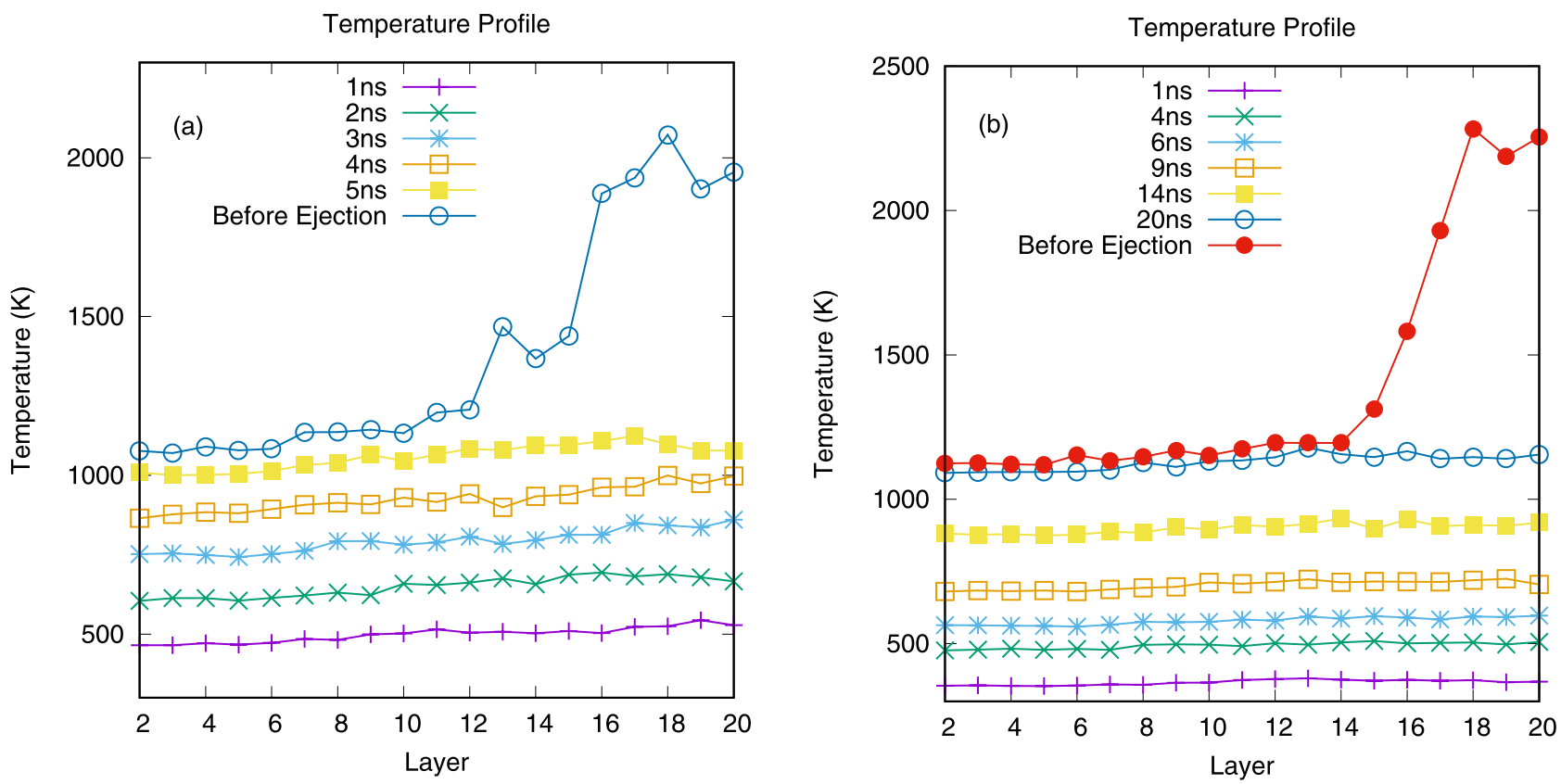

FIG. 6. Temperature profiles at different times obtained from the MD simulations. (a) Copper substrate with a $8.2 \mathrm{~nm}$ tall protrusion and a base of $8 \times 8$ lattice units and (b) a $13 \mathrm{~nm}$ tall protrusion with a base of $12 \times 12$ lattice units.

not rise as much since the bulk substrate is relatively close, and affords better heat conduction. The primary effect of elevated temperatures starting from the tip is simply enhancements in the molecular kinetic energies, leading to greater mobility and consequent lateral spreading.

For completeness, the dynamic evolution of local temperatures within the protrusion obtained from the MD simulations is discussed next. The profiles for the temperatures at different times for the $8.2 \mathrm{~nm}$ tall structure with a base of $8 \times 8$ lattice units is shown in Fig. 6(a). The $x$-axis represents the 20 grid points along the $z$-axis starting from the bottom of the substrate to the protrusion top. The temperature is predicted to increase over time, and then undergoes a much steeper rise towards the top after about $5 \mathrm{~ns}$. Physically, this is linked to the necking at the top that reduces heat flow and the effective thermal conductivity. As is well known, spatial confinement and reductions in the lateral dimensions decrease the thermal conductivity in free-standing structures as the phonon mean free path begins to approach the characteristic length scales. ${ }^{33,45}$ The reduced widths contribute to additional mini-umklapp processes and significantly increase boundary scattering. In any event, material ejection then occurs rapidly beyond this point. A similar trend is seen for the $13 \mathrm{~nm}$ tall protrusion in Fig. 6(b). The steep temperature rise near the top occurs after about $20 \mathrm{~ns}$ in this case.

Based on the above results, the following dynamic evolution can be conjectured for a nonuniform distribution of protrusions. A likely qualitative sequence might involve initial material ejection (along with possible electron emission) from the longer elements, which would gradually shorten. Others at random sites would then be recruited later in time, and the process would continue, with localized heating of the nano-array. Electron emission which could screen out the local electric fields, along with temperature increases leading to edge "rounding," would then work to eventually terminate the process.
The presence of space-charge was ignored in this analysis. Strong surface electric fields lead to increases in emission currents and give rise to charge buildup, which in turn can limit the surface electric fields. This aspect was recognized years $\mathrm{ago}^{46}$ and known to cause a transition from Fowler-Nordheim dominated current to Child-Langmuir characteristics. There have already been several elegant treatments of space-charge screening, and its role in modifying the current characteristics, especially over long time scales. ${ }^{47-50}$ However, our primary focus here is on the initial duration and the processes that collectively trigger explosive emission. Experiments have shown that the emission current from a single emitting site can be as high as $10^{8}-10^{9} \mathrm{~A} / \mathrm{cm}^{2}$, but would not substantially affect the field distributions at these initial conditions. ${ }^{51,52}$ This aspect though is likely to be an issue for field emitter arrays, ${ }^{53}$ though in the present context, such space-charge effects would likely lead to increased durations for material ejection. Finally, given the finite time required for charge buildup and the strong electrostatic forces that would pull electrons away from the emitter, the space-charge charge effects in the present simulations were neglected. However, in a broader sense, this aspect remains an important part of a very complex, dynamic, and multifacetted problem. Furthermore, details of the emitting surface geometry and any defects that may exist on (or near) the surface could also influence the emission. Both aspects can be time-dependent as material ejection proceeds. In addition, wavefunction modifications due to defects can affect the emission rates, as these would likely not be controlled by simple tunneling through a triangular barrier. Some of the quantum aspects were probed by Ang et al. ${ }^{54}$ Other important issues in this context, include the creation of desired field enhancements through mechanically stable nanostructures that rely on dissimilar length scales between nanoprotrusions and the substrate. ${ }^{55}$ 


\section{CONCLUSIONS}

A three-dimensional, time-dependent molecular dynamics model was used to study the potential for mass ejection from a metal nanoprotrusion, driven by high fields and temperature increases. The electric fields surrounding the metal emitter were calculated, and the surface distribution was used to obtain the Maxwell stress on the solid. The temperature evolution was included in a self-consistent manner.

Our results indicate that a larger aspect ratio and high local temperatures at the tip would drive the system towards mass ejection. The base area of the structure was shown to play a role as well, with longer times for ejection predicted for larger base dimensions. For example, protrusion break up times of about $25 \mathrm{~ns}$ were obtained for a $13 \mathrm{~nm}$ tall structure with a base size of $12 \times 12$ lattice units. This time scale is roughly in agreement with experimental reports on explosive emission. ${ }^{44}$ On the other hand, very short protrusions were shown to lead to a flattening which would effectively be a self-healing process and work against explosive emission. Finally, it was shown that the process involves narrowing at the nanotip region, leads to reduced thermal conductivity due to spatial confinement effects, and facilitates rapid local temperature increases.

From a practical standpoint, perhaps a simple way forward might be the experimental determination of thermal conductivities associated with such nanoprotrusions. An effort in this direction would fold in finite size effects and enable direct comparisons between MD results and experiments. Other useful efforts might include studies of material degradation in such nanostructures for determining thresholds on protrusion height, or obtaining material-dependent curves of the aspect ratio versus applied electric field, or determining the field strength-ejection duration characteristics with aspect ratio as a parameter.

\section{ACKNOWLEDGMENTS}

The authors thank S. Plimpton (Sandia National Laboratories) for the helpful discussions.

${ }^{1}$ L. M. Li, L. Liu, and J. C. Wen, J. Phys. D: Appl. Phys. 40, 5338 (2007).

${ }^{2}$ J. A. Nation, L. Schachter, F. M. Mako, L. K. Len, W. Peter, C. M. Tang, and T. S. Rao, Proc. IEEE 87, 865 (1999).

${ }^{3}$ A. I. Pushkarev and R. V. Sazonov, IEEE Trans. Plasma Sci. 37, 1901 (2009).

${ }^{4}$ S. Ya. Belomyttsev, S. D. Korovin, and I. V. Pegel', Zh. Tekh. Fiz. 69, 97 (1999) [Tech. Phys. Lett. 44, 695 (1999).

${ }^{5}$ G. A. Mesyats, JETP Lett. 57, 95 (1993).

${ }^{6} \mathrm{G}$. A. Mesyats, "The ecton and its nature, the vacuum discharge," in Pulsed Power (Springer, New York, 2005), Chap. 2, pp. 36-40.

${ }^{7}$ E. Garate, R. MacWilliams, A. Voss, D. Lovesee, K. Hendricks, T. Spencer, M. C. Clark, and A. Fisher, Rev. Sci. Instrum. 66, 2528 (1995).

${ }^{8}$ R. B. Miller, J. Appl. Phys. 84, 3880 (1998).

${ }^{9}$ G. A. Mesyats and D. I. Proskurovsky, Pulsed Electrical Discharges in Vacuum (Springer, Berlin, 1989).

${ }^{10}$ Y. E. Krasik, A. Dunaevsky, A. Krokhmal, J. Felesteiner, A. V. Gunin, I. V. Pegel, and S. D. Korovin, J. Appl. Phys. 89, 2379 (2001).

${ }^{11}$ V. Vlahos, J. H. Booske, and D. Morgan, Appl. Phys. Lett. 91, 144102 (2007).

${ }^{12}$ J. Sun, P. Wu, S. F. Huo, W. B. Tan, H. Shao, C. H. Chen, and G. Z. Liu, IEEE Trans. Plasma Sci. 42, 2179 (2014).
${ }^{13}$ E. V. Oreshkin, S. A. Barengolts, G. A. Mesyats, V. I. Oreshkin, and K. V. Khishchenko, J. Phys.: Conf. Ser. 774, 012191 (2016).

${ }^{14}$ H. Qiu, R. P. Joshi, A. Neuber, and J. Dickens, Semicond. Sci. Technol. 30, 105038 (2015).

${ }^{15}$ W. Tang, D. Shiffler, and K. L. Cartwright, J. Appl. Phys. 110, 034905 (2011).

${ }^{16}$ G. N. Fursey, M. A. Polyakov, L. A. Shirochin, and A. N. Saveliev, Appl. Surf. Sci. 215, 286 (2003).

${ }^{17}$ A. Anders, Cathodic Arcs: From Fractal Spots to Energetic Condensation (Springer-Verlag, New York, 2008).

${ }^{18}$ R. B. Miller, An Introduction to the Physics of Intense Charge Particle Beams (Plenum, New York, 1982).

${ }^{19}$ S. A. Barengolts, E. A. Litvinov, and V. G. Suvorov, IEEE Trans. Dielectrics Electr. Insul. 6, 430 (1999).

${ }^{20}$ S. Jun and L. Guozhi, IEEE Trans. Plasma Sci. 33, 1487 (2005).

${ }^{21}$ A. B. Petrin, J. Exp. Theor. Phys. 109, 314 (2009).

${ }^{22}$ S. A. Barengolts, G. A. Mesyats, and M. M. Tsventoukh, JETP 107, 1039 (2008).

${ }^{23}$ Z. Zhang, M. Giesselmann, J. Mankowski, J. Dickens, A. Neuber, and R. P. Joshi, J. Phys. D 50, 185202 (2017).

${ }^{24}$ J. M. Parson, C. F. Lynn, J. J. Mankowski, A. A. Neuber, and J. C. Dickens, IEEE Trans. Plasma Sci. 42, 3982 (2014)

${ }^{25}$ F. Djurabekova, S. Parviainen, A. Pohjonen, and K. Norlund, Phys. Rev. E 83, 026704 (2011).

${ }^{26}$ E. W. Müller and T. T. Tsong, Progress in Surface Science (Oxford, Pergamon, Oxford, 1973), Vol. 4, Part 1.

${ }^{27}$ T. T. Tsong, Surf. Sci. 70, 211 (1978).

${ }^{28}$ H. J. Kreuzer and L. C. Wang, J. de Physique 50, C8-53-C8-57 (1989).

${ }^{29}$ A. Majzoobi, R. P. Joshi, A. A. Neuber, and J. C. Dickens, AIP Adv. 5, 127237 (2015)

${ }^{30}$ J. Plateau, Mem. Inst. R. Sci. Nat. Belg. 16, 1 (1843)

${ }^{31}$ L. Rayleigh, Proc. R. Soc. London 29, 71 (1879).

${ }^{32}$ F. A. Nichols and W. W. Mullins, Trans. Met. Soc. AIME 233, 1840 (1965).

${ }^{33}$ E. Chavez-Angel, J. S. Reparaz, J. Gomis-Bresco, M. R. Wagner, J. Cuffe, B. Graczykowski, A. Shchepetov, H. Jiang, M. Prunnila, J. Ahopelto, F. Alzina, and C. M. Sotomayor-Torres, APL Mater. 2, 012113 (2014).

${ }^{34}$ A. Descoeudres, Y. Levinsen, S. Calatroni, M. Taborelli, and W. Wuensch, Phys. Rev. Spec. Top.-Accel. Beams 12, 092001 (2009).

${ }^{35}$ R. Miller, Y. Y. Lau, and J. H. Booske, J. Appl. Phys. 106, 104903 (2009).

${ }^{36}$ F. B. Hildebrand, Advanced Calculus for Applications (Prentice-Hall, Englewood Cliffs, 1962).

${ }^{37}$ S. Plimpton, J. Comput. Phys. 117, 1 (1995).

${ }^{38}$ Z. Insepov, J. H. Norem, and A. Hassanein, Phys. Rev. Spec. Top.Accel. Beams 7, 122001 (2004).

${ }^{39}$ G. Bonny, R. C. Pasianot, N. Castin, and L. Malerba, Philos. Mag. 89, 353 (2009).

${ }^{40}$ S. M. Foiles, M. I. Baskes, and M. S. Daw, Phys. Rev. B 33, 7983 (1986).

${ }^{41}$ A. Stukowski, Modell. Simul. Mater. Sci. Eng. 18, 015012 (2010).

${ }^{42}$ K. D. Rendulic and E. W. Muller, J. Appl. Phys. 38, 2070 (1967).

${ }^{43}$ C. H. Zhang, F. Kassubek, and C. A. Stafford, Phys. Rev. B 68, 165414 (2003).

${ }^{44}$ G. A. Mesyats, Herald Russ. Acad. Sci. 84, 242 (2014).

${ }^{45}$ A. Balandin and K. L. Wang, Phys. Rev. B 58, 1544 (1998).

${ }^{46}$ Y. Y. Lau, Y. Liu, and R. K. Parker, Phys. Plasmas 1, 2082 (1994).

${ }^{47}$ P. Wu and J. Sun, Phys. Plasmas 23, 013111 (2016).

${ }^{48}$ K. L. Jensen, J. Appl. Phys. 107, 014905 (2009).

${ }^{49}$ A. Rokhlenko, K. L. Jensen, and J. L. Lebowitz, J. Appl. Phys. 107, 014904 (2010)

${ }^{50}$ Y. Feng and J. P. Verboncoeur, Phys. Plasmas 12, 103301 (2005).

${ }^{51}$ S. D. Korovin, E. A. Litvinov, G. A. Mesyats, V. V. Rostov, S. N. Rukin, V. G. Shpak, and M. I. Yalandin, IEEE Trans. Plasma Sci. 34, 1771 (2006).

${ }^{52}$ F. R. Schwirzke, IEEE Trans. Plasma Sci. 19, 690 (1991).

${ }^{53}$ J. R. Harris, K. L. Jensen, J. J. Petillo, S. Maestas, W. Tang, and D. A. Shiffler, J. Appl. Phys. 121, 203303 (2017).

${ }^{54}$ L. K. Ang, W. S. Koh, Y. Y. Lau, and T. J. T. Kwan, Phys. Plasmas 13, 056701 (2006)

${ }^{55}$ T. A. de Assis and F. F. Dall'Agnol, Nanotechnology 27, 44LT01 (2016). 